\title{
TAGUNG
}

\section{Atatürks Traum - Europas Alptraum? Die europäischen Perspektiven der Türkei}

\author{
Frédéric Krumbein*
}

In seinen einleitenden Worten betonte Haluk Kabaalioğlu das Ziel der türkischen Organisatoren der Tagung, durch Forschungsprojekte zur Europäischen Union den EU-Beitritt der Türkei zu erleichtern und $\mathrm{zu}$ beschleunigen. Peter-Christian Müller-Graff erläuterte am Beispiel der französischen öffentlichen Meinung die divergierenden Positionen zu einem türkischen Beitritt.

\section{Eröffnungsrede}

Der Staatsminister und türkische Chefunterhändler Egemen Bă̌ısș für die EU-Beitrittsverhandlungen betonte die vielfältigen Vorteile für die Europäische Union, die ein türkischer Beitritt hätte und die engen Verbindungen zwischen Europa und der Türkei. Auf der internationalen Bühne sei die Türkei ein sehr aktives Land. Die Türkei ist Mitglied vieler internationaler Organisationen in Europa und Asien. Sie vermittelt zwischen Israel und seinen Nachbarstaaten und zwischen Georgien und Russland. Die Türkei besitzt die zweitgrößte Armee der NATO und spielt eine wichtige Rolle für die Verteidigung Europas. Weiterhin ist die Türkische Republik die sechstgrößte europäische Wirtschaft und ein Mitglied der G20. Im Energiesektor gibt es mit der Nabucco-Pipeline ein gemeinsames Projekt, welches von der Türkei und mehreren EU-Staaten geplant wird und die Energieversorgung Europas sichern soll. Der gemeinsam geführte Kampf gegen den Terrorismus ist ein weiteres Beispiel der erfolgreichen türkisch-europäischen Kooperation. Die Dynamik der türkischen Wirtschaft mit einem Wachstum von sieben Prozent in den letzten

\section{Turkey's EU Accession: Multi-Disciplinary Approaches}

Gemeinsame Tagung der Economic Development Foundation (IKV), der Turkish University Association for EC Studies (TUNAECS-ECSA

Turkey), des Arbeitskreises Europäische Integration e.V. (AEI-ECSA Germany) und der Yeditepe University, School of Law

Istanbul, 2./3. Oktober 2009

Opening remarks

Prof. Dr. Haluk KABAALİOĞLU, Economic

Development Foundation (IKKV), Yeditepe University, Istanbul

Prof. Dr. Dres. h.c. Peter-Christian MÜLLER-

GRAFF, Heidelberg University, Heidelberg

\section{Opening speech}

Hon. Egemen BAĞIȘ, Minister of State for EU Affairs and Chief Negotiator for EU Accession

The legal dimension

Prof. Dr. Mathias ROHE, University of ErlangenNürnberg

Prof. Dr. Tuğrul ARAT, University of Economics and Technology, Ankara

The economic dimension

Dr. Nicolaus HEINEN, Analyst, Deutsche Bank Research, Frankfurt

Prof. Dr. Nahit TÖRE, Çankaya University, Ankara

\section{The conceptual dimension}

Dr. Barbara LIPPERT, Director of Studies, Stiftung Wissenschaft und Politik (SWP), Berlin Assoc. Prof. Çiğdem NAS, IKKV, Yıldız Technical University, Istanbul

The demographic dimension

Prof. Dr. Josef SCHMID, University of Bamberg Prof. Dr. Akile GÜRSOY, Yeditepe University, Istanbul

* Frédéric Krumbein, Geschäftsführer des Arbeitskreises Europäische Integration e.V., Berlin. 
Jahren (vor der Finanzkrise) ist ebenfalls ein Argument für einen Beitritt.

Der Minister sagte, dass die Türkei sich oft von der Europäischen Union diskriminiert sähe. Die Europäische Union ist das größte Friedensprojekt der menschlichen Geschichte. Wenn ehemalige Kriegsgegner zusammenleben können, sollten sie ebenfalls mit der Türkei zusammenleben können, die nie so blutige Kriege mit europäischen Staaten geführt hat, wie die europäischen Staaten untereinander. Es ist oft schwierig den Türken zu erklären, warum der EU-Beitritt für das eigene Land so wichtig ist, wenn die Europäische Union die Beitrittskandidaten mit doppelten Standards misst.

Weiterhin betonte der Minister die Fortschritte der Türkei bei den Minderheitenrechten. Kurdische Fernsehprogramme sind inzwischen zugelassen und senden 24 Stunden am Tag. Noch vor zehn Jahren hätten Kurden Angst gehabt, zuzugeben, dass sie Kurden sind. Die Regierung trifft sich regelmäßig zu Gesprächen mit Vertretern von ethnischen und religiösen Minderheiten. Ein Drittel der Beitrittskapitel mit der Europäischen Union sind geöffnet worden. Die jetzt noch zu öffnenden Kapitel sind diejenigen, wie zum Beispiel Umwelt, die intensive und kostspielige Änderungen und Anpassungen in der Türkei erfordern. Das Hauptziel des Ministers ist es, zu gewährleisten, dass die Türkei ihre Reformen zur Anpassung an die Europäische Union weiter vorantreibt.

Schließlich verdeutlichte der Staatsminister die Bedeutung des EU-Beitritts für die Türkei und die Empfindungen auf türkischer Seite. Die Türkei ist einerseits hoffnungsvoll, da noch nie Beitrittsverhandlungen aufgenommen wurden ohne dass es zu einem Beitritt kam. Auf der anderen Seite sieht die Türkei sich oft unfair behandelt. Wenn europäische Politiker sagen, dass die Türkei einen Sonderstatus bekommen soll, ist das eine Beleidigung für die Türkei. Außerdem müssten alle 27 Mitgliedstaaten einem Sonderstatus zustimmen, weil dieser Status momentan nicht

\section{The cultural dimension}

Dr. Günter SEUFERT, Journalist and Writer, Istanbul

Prof. Dr. Nedret KURAN-BURÇOǦLU,

Yeditepe University, Istanbul

\section{The dimension of the rule of law}

Prof. Dr. Claus Dieter CLASSEN, University of Greifswald

Assoc. Prof. Dr. Sanem BAYKAL, University of Ankara

\section{The political dimension}

Dr. Heinz KRAMER, Researcher, Research Division EU External Relations, SWP, Berlin Prof. Dr. Ersin KALAYCIOĞLU, Sabancı University, Istanbul

\section{Closing remarks}

Prof. Dr. Haluk KABAALIOǦLU, İKV, Yeditepe University, Istanbul

Prof. Dr. Dres. h.c. Peter-Christian MÜLLER-

GRAFF, Heidelberg University, Heidelberg

existiert und alle Staaten für die Aufnahme von Beitrittsverhandlungen waren.

\section{Die rechtliche Dimension}

Mathias Rohe hat über die rechtliche Dimension des Beitritts der Türkei zur Europäischen Union gesprochen. Er verglich das Recht mit den Säulen der Brücke, welche zwischen der Europäischen Union und der Türkei gebaut werden könnte.

Grundsätzlich kann jeder europäische Staat, der die Beitrittskriterien der Europäischen Union erfüllt, Mitglied der Europäischen Union werden. Die Türkei ist ein europäischer Staat. Dieses Kriterium ist erfüllt, da Beitrittsverhandlungen aufgenommen wurden. In den 1980er Jahren wurde beispielsweise Marokkos Antrag sofort abgelehnt.

Welche Hindernisse gibt es für eine Aufnahme der Türkei? Auf der europäischen Seite besteht die Angst, dass jede weitere Erweiterung die Europäische Union als Projekt des Friedens gefährden könnte. Die Europäische Union ist ein einzigartig erfolgreiches Projekt für die Erhaltung des Friedens in Europa. Es gibt in Deutschland nach Jesu Geburt keine vergleichbar lange Periode ohne einen 
Krieg. Das gleiche gilt für Frankreich und für andere EU-Staaten. Dieser Erfolg der Europäischen Union begründet die Angst vor einer Aufnahme der Türkei. Diese europäischen Ängste müssen bei der Diskussion der europäischen Perspektiven der Türkei berücksichtigt werden. Weiterhin dürfen aus europäischer Sicht bei der Aufnahme der Türkei nicht die gleichen Fehler gemacht werden wie bei der Aufnahme von Rumänien, Bulgarien und Zypern. Rumänien und Bulgarien erfüllen die Kopenhagener Kriterien nicht und Zypern hat einen ungelösten internen Konflikt.

Viele EU-Bürger sind gegen einen türkischen EU-Beitritt wegen der türkischen Menschenrechtsbilanz. Der jüngste EU-Fortschrittsbericht zur Türkei bemängelt unter anderem, dass der türkische Oberste Gerichtshof fast die Regierungspartei AKP wegen einer vergleichsweise unbedeutenden Angelegenheit verboten hätte. Die Rolle des Militärs im politischen System der Türkei ist ein weiteres Problem für einen demokratischen Staat. Im Strafgesetzbuch sind menschenrechtlich bedenkliche Paragrafen enthalten. So müssen Journalisten mit staatlicher Repression rechnen, wenn sie über politisch sensible Fragen, wie den Völkermord an den Armeniern, schreiben. Die Religionsfreiheit ist ebenfalls eingeschränkt. Christen und Aleviten werden in ihrer Religionsausübung behindert. Viele Europäer sind auch gegen einen Beitritt der Türkei aufgrund der islamischen Identität der Türkischen Republik. Dabei wird übersehen, dass der Islam eine sehr vielfältige Religion ist. Der türkische Islam beispielsweise ist ein ganz anderer als der fundamentalistische Islam in Saudi-Arabien. Kein einziges der Kopenhagener Kriterien schreibt die Religion des Beitrittskandidaten vor. Im Übrigen hat die Europäische Union bereits dreißig Millionen muslimische Bürger.

Auf der türkischen Seite fehlt das Vertrauen in die Europäische Union. Viele Türken fürchten, dass diese den Vertrag von Sèvres wiederholen wollen. Im Vertrag von Sèvres wurden der Türkei nach dem Ersten Welt- krieg schwere Lasten aufgebürdet und das Osmanische Reich als staatliche Einheit zerschlagen. Viele Türken unterstellen der Europäischen Union, dass diese die Minderheiten in der Türkei stärken und damit die nationale Einheit der Türkei untergraben möchte. Diese Angst ist unbegründet. Es gibt kein Interesse an einer Schwächung der Türkei. Niemand in der Europäischen Union will eine instabile Türkische Republik in dieser fragilen Weltregion. So wie die Türken in Europa ihre Religion unbehindert ausüben können, setzt sich die Europäische Union für die Rechte religiöser Minderheiten in der Türkei ein.

Drei Punkte bildeten den Abschluss des Vortrags von Rohe. Erstens, beim europäisch-türkischen Dialog spielt die unterschiedliche Debattenkultur zwischen vielen Europäern (insbesondere Deutschen) und den Türken und Muslimen eine wichtige Rolle. Deutsche sind sehr direkt und direkte Kritik wird oft als Beleidigung empfunden. Umgekehrt sprechen Türken Differenzen nur indirekt an, um das Gesicht des Gegenübers zu wahren. Europäer betrachten dieses Diskussionsverhalten als ineffektiv. Diese unterschiedliche Dialogkultur muss beachtet und respektiert werden. Zweitens, die Europäische Union hat die Verhandlungen über einen Beitritt der Türkei zur Europäischen Union begonnen und muss seine daraus resultierenden Verpflichtungen ernst nehmen. Alles andere wäre eine Beleidigung für die Türkei. Die sogenannte, privilegierte Partnerschaft ${ }^{*}$ als Ersatz einer Vollmitgliedschaft ist kein ausgereiftes Konzept. Niemand weiß, was mit einer , privilegierten Partnerschaft' gemeint ist und worin diese Partnerschaft bestehen könnte. Drittens, die Türkei sollte die von der Europäischen Union geforderten Reformen für ihr eigenes Wohl und nicht für den angestrebten EU-Beitritt durchführen.

Kabaalioğlu kommentierte den Vortrag von Rohe. Er betonte, dass die Türkei bereits 1963 ein Assoziierungsabkommen mit der Europäischen Wirtschaftsgemeinschaft (EWG) abge- 
schlossen hat. Das Ziel dieses Abkommens war die Vollmitgliedschaft. Das Abkommen sah mehrere Stufen der Freizügigkeit von Waren und Personen vor. Inzwischen hat die Türkei eine vollständige Zollunion mit der Europäischen Union und eine Volkswirtschaft, die auf dem EU-Binnenmarkt konkurrieren kann. Weiterhin betonte Kabaalioğlu, dass die Türkische Republik bereits unter Atatürk viele fortschrittliche Reformen durchgeführt hat, wie eine Demokratisierung des Landes und Maßnahmen für die Gleichberechtigung der Frau.

Zur Zypernfrage sagte er, dass internationale Abkommen eine Mitgliedschaft Zyperns in internationalen Organisationen verböten, wenn nicht gleichzeitig Griechenland und die Türkei der gleichen Organisation beiträten. Der EU-Beitritt Zyperns war nur möglich, weil die Europäische Union sich selbst als supranationale und nicht als internationale Organisation definierte. Die Kopenhagener Beitrittskriterien schreiben vor, dass ein Land keine Grenzstreitigkeiten haben darf. Zypern hat auf seinem Territorium eine UN-Friedenstruppe stationiert.

Viele Türken fühlen sich durch die Europäische Union bei der Visavergabe diskriminiert. So müssen türkische Geschäftsleute, um ein Visum zu erhalten, ihre Bankdaten angeben und eine Einladung vorweisen. Außerdem müssen sie oft Monate auf ein Visum warten.

\section{Die wirtschaftliche Dimension}

Nicolaus Heinen hält weitere Reformen im wirtschaftlichen Bereich für notwendig, um das volle Potenzial der türkischen Wirtschaft ausschöpfen zu können. Die öffentlichen Finanzen der Türkei sind durch die globale Wirtschaftskrise in einer schlechten Lage. Voraussichtlich gibt es 2009 ein Budgetdefizit von 6,3 Prozent des Bruttoinlandsprodukts. Die türkische Zentralbank ist nicht vollständig unabhängig. Sie sollte von der Regierung unbeeinflusst agieren können. Die Privatisierungspolitik der Türkei sollte eben- falls vorangetrieben werden. Reformen im Energie- und Agrarsektor wären weitere Schritte für eine Erhöhung der türkischen Wettbewerbsfähigkeit. Mittelfristig werden hohe wirtschaftliche Kosten für die Türkei bei der Umsetzung des ,acquis communautaire', wie bei der Implementierung des EU-Umweltrechts, entstehen. Diese Kosten könnten das Wachstum der Türkei hemmen, falls die Türkei zu früh der Europäischen Union beitritt. Langfristig könnte ein ,brain drain“ ein Problem für die Türkei darstellen. Viele junge, gut ausgebildete Türken könnten in die Europäische Union streben und würden dann in der Türkei als Fachkräfte fehlen. Die Hauptaufgabe der türkischen Wirtschaft ist der Umbau zu einer Volkswirtschaft mit hochwertigen Waren, die über die Qualität konkurrieren können. Momentan konkurriert die türkische Volkswirtschaft vornehmlich über den Preis.

Die Europäische Union sollte sich aber ebenfalls reformieren. Das Budget der Europäischen Union müsste geändert werden. Bei einer Aufnahme in die Europäische Union würden jährlich etliche Milliarden Euro als Strukturhilfen und Agrarsubventionen in die Türkei fließen. Diese Summe kann die Europäische Union nicht schultern. Weitere Reformen sind damit sowohl in der Türkei als auch in der Europäischen Union notwendig, um den EU-Beitritt der Türkei erfolgreich zu bewältigen.

Zum Abschluss seines Vortrags zitierte Heinen Charles de Gaulle, der ein religiöses Argument gegen Großbritanniens Beitritt zur Europäischen Gemeinschaft vorbrachte: „Die Briten haben eine andere Religion als wir". Meinungen können sich ändern.

Nahit Töre referierte anschließend über die türkische Sicht auf die wirtschaftliche Dimension eines EU-Beitritts. Die türkische Wirtschaft ist bereits seit langer Zeit auf Europa ausgerichtet. 1923 waren die wichtigsten Handelspartner der Türkei sieben europäische Länder, von denen sechs später die EU-Grün- 
dungsstaaten waren. Die Haupthandelspartner der Türkei sind heutzutage ebenfalls europäische Staaten, zuvorderst Deutschland. 50 Prozent der türkischen Exporte fließen in die Europäische Union. Seit Einführung der Zollunion zwischen der Türkei und der Europäischen Union 1996 haben beide Volkswirtschaften von der Zollunion profitiert. Der türkische Export von technologischen Gütern, wie Fernsehern, Kühlschränken und Autos, hat sich seit Beginn der Zollunion erheblich gesteigert. Die Wettbewerbsfähigkeit, die Direktinvestitionen und die Zahl der Touristen haben zugenommen.

Freihandelsabkommen der Europäischen Union mit Drittstaaten sind ein Problem aus türkischer Sicht, da die Türkei aufgrund der Zollunion automatisch alle Handelsabkommen übernehmen muss, welche die Europäische Union mit Drittstaaten unterzeichnet. Die Zunahme der Handelsabkommen zwischen der Europäischen Union und Drittstaaten verschlechtert die Wettbewerbsfähigkeit der Türkei, weil die Waren aus Drittstaaten ohne ein bestehendes bilaterales Handelsabkommen über den Umweg der Europäischen Union in die Türkei kommen, ohne dass Zölle erhoben werden können. Die Europäische Union sollte deshalb die Türkei bei Verhandlungen mit Drittstaaten beteiligen und Druck auf die Drittstaaten ausüben, dass diese zeitgleich mit der Europäischen Union auch mit der Türkei ein Handelsabkommen abschließen. Insgesamt ist die Zollunion aber profitabel für die türkische Wirtschaft.

\section{Die konzeptuelle Dimension}

Was ist das Problem der türkisch-europäischen Verhandlungen? Dies war die zentrale Fragestellung des Vortrags von Barbara Lippert. Im Regelfall ist das Ziel von Beitrittsverhandlungen der Beitritt des Landes zur Europäischen Union. Ein Beitrittskandidat muss für eine Aufnahme in die Europäische Union die Kopenhagener Kriterien erfüllen und sein Gewicht in der Europäischen Union verhandeln.
Die Türkei ist kein gewöhnlicher Beitrittskandidat. Diese türkische Sonderstellung aus europäischer Sicht ergibt sich aus den vielfältigen Vorbehalten in der Europäischen Union gegenüber einem Beitritt. Die Aufnahme der Türkei in die Europäische Union hätte weitreichende Konsequenzen für die Gestaltung der EU-Außenpolitik und für das innere Machtgefüge der Europäischen Union. Ein Beitritt wäre in seinen Auswirkungen vergleichbar mit dem Beitritt Großbritanniens 1973.

Das offizielle Ziel der europäisch-türkischen Verhandlungen ist ein Beitritt der Türkei. Durch die europäischen Vorbehalte bleibt ein Beitritt aber unsicher. Eine vollständige Mitgliedschaft der Türkei könnte für die Europäische Union finanziell und politisch zu kostspielig werden. Welche Optionen einer EUMitgliedschaft beziehungsweise einer engen Kooperation außerhalb einer Vollmitgliedschaft gibt es? Erstens, es gibt Vollmitgliedschaften von Staaten, die den, acquis communautaire nicht vollständig umsetzen. Beispiele dafür sind Bulgarien und Rumänien. Zweitens, es gibt Abstufungen in der Mitgliedschaft. So gibt es ,opt-outs ' in bestimmten Bereichen der Integration, wie beim Euro oder dem Schengenraum. Beispiele dafür sind Schweden und Großbritannien. Eine ,reduzierte" oder ,zweitklassige" Mitgliedschaft mit verschiedenen aufgezwungenen ,opt-outs‘ wäre eine Möglichkeit für einen türkischen Beitritt. Es ist aber unsicher, ob die Türkei solch eine, zweitklassige“ Mitgliedschaft überhaupt akzeptieren würde. Drittens, es gibt Länder wie Norwegen und Island, die Mitglieder des Europäischen Wirtschaftsraums sind, ohne Mitglieder der Europäischen Union zu sein. Das wäre vermutlich keine Möglichkeit für die Türkei, weil die Europäische Union der Türkei keine Freizügigkeit im Personenverkehr einräumen würde. Viertens gibt es die Möglichkeit von verschiedenen Stufen der Assoziation mit der Europäischen Union ohne eine Mitgliedschaft. Assoziierungsabkommen können dabei von Freihandel plus ,ein Prozent' bis Mitgliedschaft mi- 
nus ,ein Prozent" reichen (Walter Hallstein). Es gibt also eine große Bandbreite von Möglichkeiten bei Assoziierungsabkommen. Eine Mitgliedschaft würde aber eine vollständige Partizipation an Entscheidungsprozessen bedeuten. Diese Partizipation existiert bei Assoziierungsabkommen nicht. Fünftens wäre eine multilaterale und projektorientierte Kooperation, wie die Mittelmeerunion, denkbar.

Neben diesen bereits vorhandenen Optionen sind neue Konzepte von EU-Mitgliedschaften notwendig. So schlägt beispielsweise Andreas Maurer „modulierte Mitgliedschaften“ vor. So gäbe es die Möglichkeit in bestimmten Politikbereichen der Europäischen Union Mitglied zu sein und in anderen nicht. Das Ergebnis wäre eine sehr komplexe Europäische Union, die auf der Grundlage der bestehenden Verträge so nicht konstruiert werden könnte. In den EU-Gremien wäre die Mitsprache des ,modulierten Mitglieds' auf bestimmte Politikbereiche begrenzt. Ein solches Modell ist politisch schwierig umzusetzen. Es gibt grundsätzlich wenig politische Anreize, Mitsprache in bestimmten Bereichen aufzugeben. So hat selbst Tschechien kein Interesse daran, aus der EU-Fischereipolitik auszusteigen. Mitsprache ist immer positiv und bietet Verhandlungsmasse bei anderen Verhandlungen, die für Tschechien wichtiger sind. Schließlich gibt es das kaum ausgearbeitete Konzept der ,privilegierten Partnerschaft'. Dieses Konzept wird in Deutschland zunehmend weniger verwendet, aber es ist nach wie vor lebendig und beinhaltet eine immer engere Integration bis hin zu einem Beobachterstatus in der Europäischen Union.

Solange die Türkei auf ihrem Status als Beitrittsland besteht, ist es aber fast unmöglich eine andere Option anzubieten. Erst wenn die Türkei ein anderes Konzept als eine Vollmitgliedschaft akzeptieren würde, könnte eine Alternative entwickelt werden. Eine breitere und tiefere Kooperation zwischen der Türkei und der Europäischen Union bleibt ohne eine Vollmitgliedschaft der Türkei grundsätzlich möglich.
Çiğdem Nas sprach von der Konditionalität als Instrument der europäischen Beitrittspolitik. Die Europäische Union nutzt, wie andere multilaterale Organisationen, Konditionalität als Instrument der Beitrittspolitik. Anreize und Sanktionen werden verwendet, um Beitrittsländer dazu zu bringen, die Voraussetzungen einer Mitgliedschaft zu erfüllen. So müssen Beitrittskandidaten den , acquis communautaire' implementieren, bevor sie Mitglieder werden können. Die Europäische Union beeinflusst über das Instrument der Konditionalität die Innenpolitik von Staaten und kann Wandel herbeiführen. Bestimmte Kräfte in einem Land werden gestärkt. Bei der Konditionalität besteht aber immer die Gefahr, dass beispielsweise Demokratisierung und die Umsetzung von Menschenrechten nur oberflächlich stattfinden und nicht substanziell und dauerhaft sind. Der entscheidende Anreiz bleibt eine Vollmitgliedschaft mit umfassender Beteiligung an allen politischen Entscheidungsprozessen und mit der Gewährung finanzieller Hilfen durch die Europäische Union. Die Politik der Konditionalität kann nur funktionieren, wenn die Mitgliedschaft eine realistische Möglichkeit bleibt. Wenn diese Option nicht mehr glaubwürdig erscheint, ist die Politik zum Scheitern verurteilt. Es besteht kein ausreichender Anreiz mehr, weitere Reformen durchzuführen. Das Kapitel zur Sozialpolitik in den türkisch-europäischen Beitrittsverhandlungen ist ein Beispiel. Es wurde von der Türkei bislang nicht geöffnet, weil die Kosten der Umsetzung des EU-Rechts in diesem Bereich für die türkische Wirtschaft hoch sind. Entsprechend zögert die Regierung, weil die Mitgliedschaft in der Europäischen Union unsicher ist und die Türkei eventuell keine Gegenleistung für die zu zahlenden Kosten der Umsetzung des EUSozialrechts erhält.

Falls die Europäische Union die Vollmitgliedschaft der Türkei ablehnt, obwohl die Europäische Union Verhandlungen mit der Türkischen Republik aufgenommen hat, könnte das internationale Image der Europäischen Union Schaden nehmen. 
In der anschließenden Diskussion betonte $\mathrm{Ka}$ baalioğlu noch einmal, dass keine türkische Regierung eine ,privilegierte Partnerschaft ${ }^{6}$ akzeptieren und ein solches Angebot als Beleidigung ansehen würde. Das Verhältnis der Türkei zur Europäischen Union würde sich deutlich verschlechtern, falls die Europäische Union der Türkei etwas anderes als eine Vollmitgliedschaft anbieten würde. Die Vorstellung, dass die Türkische Republik in einigen Bereichen der EU-Politik sehr gut kooperieren würde ohne die Aussicht auf volle Mitsprache, ist unrealistisch.

Müller-Graff unterstrich, dass die entscheidende Hürde für eine EU-Mitgliedschaft der Türkei die dann entstehende neue Machtbalance innerhalb der Europäischen Union wäre. Aus diesem Grund ist Frankreich gegen eine Vollmitgliedschaft. Lippert ergänzte, dass sich das Dreieck Frankreich-DeutschlandGroßbritannien ändern müsste. Natürlich denken die EU-Staaten darüber nach, was bei einem EU-Beitritt der Türkei ihre zukünftige Rolle innerhalb der Union wäre. Über die neue Gestalt der Europäischen Union mit der Türkei als Mitglied muss diskutiert werden. Diese Diskussion wäre besser vor Beginn der türkischen Beitrittsverhandlungen geführt worden. Sie bleibt aber auch nach Beginn der Verhandlungen unvermeidlich.

\section{Die demografische Dimension}

Wann das Wachstum der türkischen Bevölkerung zu einem Ende kommen wird, war die leitende Frage des Vortrags von Josef Schmid. Die Türkei hat momentan 74 Millionen Einwohner. Bei Gründung der Republik hatte die Türkei nur 13 Millionen Einwohner. Zurzeit beträgt das jährliche Wachstum der Bevölkerung 1,2 Prozent. Vermutlich wird die Türkei erst Mitte des 21. Jahrhunderts den Höhepunkt ihrer Einwohnerzahl erreichen, weil die Wachstumsrate erst seit kurzer Zeit sinkt. Im Vergleich zur Türkei ist in der Mehrheit der europäischen Staaten die Zahl der jährlichen Toten höher als die Zahl der Geburten. Frank- reich und Großbritannien stellen mit relativ hohen Geburtenraten Ausnahmen dar.

Die türkische Bevölkerung wird nach Prognosen voraussichtlich 2025 87,4 Millionen und 2050 97,4 Millionen umfassen. In Deutschland wird die Einwohnerzahl 2025 nur noch 79,6 Millionen und 2050 71,4 Millionen betragen. Die Fertilitätsrate beträgt in Deutschland 1,3 und in der Türkei 2,1 (Werte von 2005-2010). Diese Prognosen sind nur eine Fortführung der aktuell bestehenden Dynamiken. Die Türkei befindet sich im Prozess der demografischen Transition. Der Begriff demografische Transition meint eine Veränderung im Wachstum der Bevölkerung bedingt durch neue Familienstrukturen und neue Konsum- und Lebensweisen. Im Rahmen der gesellschaftlichen Modernisierung entwickeln sich die Gesellschaften alle nach und nach hin zu einer demografischen Transition mit niedrigen Geburtenraten. Entscheidend bei der Dynamik des türkischen Bevölkerungswachstums ist aber in erster Linie das Heranwachsen der Eltern aus der jungen Generation und nicht die Zahl der Kinder in den Familien. Die Zahl der Kinder in den Familien nimmt ab, aber die Zahl der Eltern nimmt noch weiter zu. Dieser Faktor kompensiert teilweise den Rückgang der Geburtenrate.

Die Europäische Union wird zwischen 2020 und 2030 eine große Herausforderung zu bewältigen haben, weil dann viele der ,Babyboomer' aus den Nachkriegsjahren in Rente gehen werden und gleichzeitig eine zahlenmäßig schwache Generation von jungen Menschen diese Rentner wird unterstützen müssen. Die europäische Arbeitsbevölkerung mit einem hohen Durchschnittsalter könnte sich in einer modernen Welt möglicherweise als weniger anpassungsfähig erweisen als eine junge Arbeitsbevölkerung.

Eine große Herausforderung in der Türkei stellt die Integration der zahlenmäßig großen jungen Bevölkerung in den Arbeitsmarkt dar. 
Akile Gürsoy referierte über die Veränderungen der Lebensbedingungen in der Türkei und ihre Auswirkungen auf die türkische Demografie. Die Türkei weist eine sehr geringe Scheidungsrate auf. Fast jeder Türke ist verheiratet. Es gibt nur wenige Singles. Viele Türken heiraten aber später als früher. Die Familienstruktur in der Türkei ändert sich zunehmend, aber die Familie bleibt sehr wichtig. Familienbeziehungen und ein System der Unterstützung zwischen den Generationen sind zentrale türkische Werte. Die soziale Mobilität ist sehr hoch.

Die durchschnittliche Lebenserwartung in der Türkei in den 1920er Jahren betrug nur dreiBig Jahre. Die Kinder- und Müttersterblichkeit waren in den Anfangsjahren der Türkischen Republik sehr hoch. Die Rate der Kindersterblichkeit war 350 auf 1.000 Geburten. Heute beträgt die Lebenserwartung in der Türkei über siebzig Jahre. Seit drei Jahren muss die Türkei keine neuen Grundschulen mehr bauen, weil die Zahl der jungen Türken abnimmt. Die Kindersterblichkeit ist deutlich zurückgegangen und die typischen Todesarten in der Türkei sind mit denen in Westeuropa vergleichbar, wie zum Beispiel Krebs sowie Herz- und Kreislauferkrankungen.

\section{Die kulturelle Dimension}

Günter Seufert betonte, dass die kulturellen Herausforderungen eines türkischen EU-Beitritts sehr viel stärker von Konzepten, wie Identität, abhängen. Im Bereich der Kultur gibt es oftmals vereinfachte Darstellungen von geschichtlichen Ereignissen und Kulturen. Der Islam wird im Westen oft negativ dargestellt und seit den Anschlägen von alQaida werden Islam und Gewalt oft gleichgesetzt. In Europa betrachten viele die Türkei nicht als Teil der europäischen Kultur. Eine abschließende Antwort auf die Frage, was Teil Europas und was Teil der europäischen Kultur ist, existiert aber nicht. Der Kulturbegriff ist schier unendlich. Der Westen sieht seine eigene Geschichte als einen Weg des ewigen Fortschritts und blendet dabei Ent- wicklungen wie Rassismus, Kolonialismus und den Holocaust aus.

Die Reformdebatte in der Türkei wird von Ängsten um die Einheit der Türkei geprägt. Viele Türken fürchten, dass Reformen im Rahmen des EU-Beitrittsprozesses die Einheit des Staates gefährden könnten, indem die Minderheiten zu viele Rechte bekommen. Der EU-Beitritt der Türkei wird hingegen von ethnischen und religiösen Minderheiten in der Türkei als Garantie ihrer Rechte angesehen. Die Frage der kulturellen Rechte, das heißt die Gewährung der kulturellen und religiösen Freiheit für Kurden, Aleviten und andere Minderheiten, ist zentral für die aktuelle politische Diskussion in der Türkei.

Konzepte von Staatsbürgerschaft und Nation sind ebenfalls wichtig. Staatsbürgerschaft meint die Zugehörigkeit des Einzelnen zur Nation und steht für politische Rechte. Hinter diesem Konzept verbergen sich Demokratie und Menschenrechte. Der französische Nationalismus ist universell und multikulturell. Jeder kann der französischen Nation zugehörig sein, er muss sich nur den Werten der Französischen Republik verpflichtet fühlen. Der deutsche Nationalismus ist hingegen partikularistisch und ethnisch gebunden. Aufgrund dieser Tradition hat Deutschland bis heute ein Staatsbürgerschaftsrecht, welches vielen Ausländern das Recht auf eine Staatsbürgerschaft verwehrt. Der türkische Nationalismus ist eine Mischung aus dem französischen und deutschen Konzept. Er ist einerseits universell, andererseits ethnisch. Die Türken sind sunnitische Muslime und bilden damit eine einheitliche Kultur. Gleichzeitig verfügt der türkische Nationalismus über die universellen und säkularen Werte der Türkischen Republik. Minderheiten, wie die Kurden oder die Aleviten, leiden unter dem partikularistischen Konzept einer Ethnie.

Nedret Kuran-Burçoğlu sprach über soziokulturelle Probleme, die bei einer Aufnahme der Türkei in die Europäische Union auftreten könnten. So bestehen auf europäischer Seite 
Vorbehalte wegen wahrgenommener kultureller Differenzen und Vorurteile über die Türken. Die Türken werden oft als Gastarbeiter betrachtet oder als , die Anderen“. Das Gastarbeiterbild in Deutschland entstand in den 1960er und 1970er Jahren und das Bild ,der Anderen" in der Zeit des Osmanischen Reiches als die Türken weite Teile Europas beherrschten. In Folge des 11. September 2001 kommt eine zunehmende Islamophobie hinzu. Weiterhin prägen der Türkei skeptisch bis feindlich gesinnte Lobbies die europäische Wahrnehmung der Türkei. So verbreiten die armenische, griechische und kurdische Lobby im Westen ein eher negatives Türkeibild.

Auf der türkischen Seite bestehen ebenfalls Vorbehalte aufgrund wahrgenommener kultureller Differenzen. Die Türken haben Angst vor einem Verlust der eigenen Identität und Souveränität. Es gibt einen verbreiteten Euroskeptizismus und die Bereitschaft, die europäischen Werte zu übernehmen, ist nicht ausgeprägt.

Zum Abbau dieser Vorurteile und Vorbehalte sollten die gemeinsamen Interessen der Europäischen Union und der Türkei näher spezifiziert werden. Die kulturelle Vielfalt sollte als Wert anerkannt und geschätzt werden. Gemeinsame Projekte, wie der Austausch von Studenten, sollten vorangetrieben werden.

\section{Die rechtsstaatliche Dimension}

Claus Dieter Classen referierte über Fortschritte und Defizite der Türkei im rechtsstaatlichen Bereich. Die Europäische Union verfügt über fundamentale gemeinsame Werte, wie Freiheit, Demokratie und Rechtsstaat. Diese Werte müssen von jedem Mitgliedsland respektiert werden. Rechtsstaatlichkeit meint das Regieren durch anerkannte und klare rechtliche Regeln, die durch die Legislative verabschiedet wurden.

Die Fortschrittsberichte der Europäischen Union zur Türkei sehen mehrere Probleme im Bereich der Menschenrechte. Der Europäische Gerichtshof für Menschenrechte hat die
Türkei öfter verurteilt als andere Staaten in Europa. Hinzu kommt, dass diese Verurteilungen oft in schwerwiegenden Fällen erfolgt sind, wie beispielsweise Verstöße gegen das Folterverbot. Menschenrechte sind ein zentraler Faktor zur Beurteilung der möglichen Mitgliedschaft eines Landes in der Europäischen Union. Die Statistiken des Europäischen Gerichtshofes für Menschenrechte zeigen, dass die Türkei oftmals fundamentale Menschenrechte, wie das Folterverbot oder, habeas corpus', verletzt. 20 der 45 Verurteilungen zu Folterfällen des Europäischen Gerichtshofes für Menschenrechte betreffen die Türkei, nur zwei die Mitgliedstaaten der Europäischen Union. Die Fortschrittsberichte der Europäischen Union zeigen Verbesserungen in der Menschenrechtssituation. Trotzdem bestehen weiterhin Probleme. Die Unterzeichnung des Zusatzprotokolls der UN-Antifolterkonvention durch die Türkei könnte ein wichtiger Schritt in die richtige Richtung sein. Dieses Zusatzprotokoll muss allerdings noch vom türkischen Parlament ratifiziert werden.

In der Türkei existiert ein Gesetz zum Schutz der türkischen Identität. Ein solches Gesetz behindert die Meinungsfreiheit und hält viele Menschen davon ab, ihre Meinung zu sagen. Der Gebrauch anderer Sprachen als der türkischen und die Ausübung anderer Religionen als der islamischen muss gewährleistet werden. Hier bestehen ebenfalls einige Probleme.

Die Türkei hat insgesamt substanzielle Fortschritte hin $\mathrm{zu}$ einem funktionierenden Rechtsstaat gemacht. Aber viele Reformen müssen noch umgesetzt werden, bevor die Türkei reif für einen Beitritt zur Europäischen Union ist. Die institutionelle Umsetzung des Rechts in der Türkei muss gestärkt werden, unter anderem durch eine bessere Aus- und Fortbildung der Verwaltung und die Bekämpfung der Korruption. Die Rolle des Militärs muss beschränkt werden. Beamte müssen denselben Gesetzen unterliegen wie zivile Personen. Die Unabhängigkeit der Gerichte muss ebenfalls verbessert werden. 
Sanem Baykal unterstrich in ihrem Vortrag die Einzigartigkeit der europäischen Integration mit ihren Prinzipien Freiheit, Demokratie und Rechtsstaat. Das Prinzip des Rechtsstaats ist komplementär zu Demokratie und Menschenrechten. Es ist als Prinzip schwer zu definieren und umstritten in seinem Inhalt. Der Europäische Gerichtshof hat nie versucht dieses Konzept zu definieren. Er hat es nur in seinen Urteilen angewendet. Das Konzept des Rechtsstaats beinhaltet eine lange Liste von Kriterien, wie Gleichheit vor dem Gesetz, individuelle Rechte, Legalität, die Möglichkeit gegen Verwaltungsakte zu klagen etc. Rechtsstaatlichkeit ist ein zu erfüllendes Kriterium für die Mitgliedstaaten und Beitrittskandidaten. Das Konzept des Rechtsstaats sollte aber besser definiert werden, damit Türken der Europäischen Union nicht die Anwendung doppelter Standards vorwerfen können.

Hindernisse auf dem Weg zu mehr Rechtsstaatlichkeit in der Türkei sind eine lange Tradition eines starken Staates und einer machtvollen Administration. Liberale Werte werden erst seit einigen Jahren intensiv diskutiert und die Tradition des starken Staates ist schwer zu ändern. Die Türkei wird die notwendigen liberalen Reformen nach Ansicht von Baykal durchführen, aber es wird seine Zeit brauchen. Die Europäische Union sollte der Türkei bei der Durchführung dieser Reformen mehr helfen.

\section{Die politische Dimension}

Heinz Kramer hat über einige Aspekte der politischen Dimension eines türkischen EU-Beitritts gesprochen. Als erstes hat er die europäischen Perspektiven der Türkei historisch eingeordnet. Der Weg zur Vollmitgliedschaft der Türkei begann bereits mit der Unterzeichnung des Assoziierungsabkommens zwischen der EWG und der Türkei 1963. Kein anderes Land hat so lange auf die Perspektive einer Vollmitgliedschaft warten müssen wie die Türkei. Das lag aber nicht nur an der Europäischen Union, sondern auch an verschiedenen türkischen Regierungen. Einzig bei der
Türkei wurde von Beginn an der europäische Charakter des Landes immer wieder infrage gestellt. Das Zitat von Walter Hallstein bei der Unterzeichnung des Assoziierungsabkommens, dass die Türkei damit ihre europäische Perspektive zum Ausdruck bringe, ist im Kontext des Kalten Krieges zu betrachten. Die Türkei wurde während des Kalten Krieges als Teil des freien Europas angesehen.

Anschließend sprach Kramer über die Meinung der europäischen Öffentlichkeit. In Meinungsumfragen („Eurobarometer") sind 46 Prozent der Europäer gegen jede weitere EUErweiterung. 55 Prozent der Europäer sind gegen eine Mitgliedschaft der Türkei, nur 31 Prozent dafür. In 14 Mitgliedstaaten sind Mehrheiten der Bevölkerung für einen türkischen Beitritt. In 13 Staaten sind Mehrheiten dagegen. Auf der Regierungsebene sind nur wenige Regierungen offen gegen eine Mitgliedschaft der Türkei. Allerdings sprechen sich auch nur wenige Regierungen nachdrücklich für eine Mitgliedschaft aus. Viele protürkische Regierungen sind aus politischen Gründen mit öffentlichen Stellungnahmen zugunsten eines Beitritts zurückhaltend. Der französische Präsident Nicolas Sarkozy dominiert die Wahrnehmung der EU-Position zur Aufnahme der Türkei, weil niemand eine protürkische Kampagne initiiert und durchführt. Die meisten Gegner sehen bei einer Aufnahme der Türkei die europäische Identität in Gefahr. Das Scheitern des Vertrags über eine Verfassung für Europa und die Machtübernahme von Nicolas Sarkozy und Angela Merkel haben die Gegner einer Türkeimitgliedschaft an Kraft gewinnen lassen. In der Türkei sind deshalb viele skeptisch, ob die Europäische Union $\mathrm{zu}$ ihren Verpflichtungen im Rahmen der Beitrittsverhandlungen stehen wird. Präsident Nicolas Sarkozy verletzt nach Ansicht von Kramer die politische Solidarität unter den EU-Mitgliedstaaten, weil er die Eröffnung einiger Kapitel bei den Verhandlungen blockiert. Diese Blockade widerspricht den Beschlüssen des Europäischen Rates, der die Beitrittsverhandlungen mit der Türkei mit dem Ziel des Beitritts beschlossen hat. 
Die Überwindung der zypriotischen Teilung ist ein weiteres Hindernis für einen türkischen EU-Beitritt. Zypern kann eine Mitgliedschaft der Türkei blockieren. Deshalb wird Zypern eine Vereinigung der Insel zu seinen Konditionen anstreben. Dies wiederum wird für die türkische Seite vermutlich nicht akzeptabel sein.

Das europäische Ziel einer starken und effektiven Gemeinsamen Außen- und Sicherheitspolitik wiederum stärkt die Beitrittsposition der Türkei. Von Anfang an war die Kooperation im Sicherheits- und Verteidigungsbereich ein entscheidendes Element in den türkischwestlichen Beziehungen. Im Kalten Krieg war die Türkei ein wichtiger Verbündeter. Selbst die Gegner eines türkischen Beitritts sehen die Türkei als einen verlässlichen und entscheidenden Verbündeten im Bereich der Sicherheitspolitik an.

In der Türkei selbst hat die Regierung unter Ministerpräsident Recep Tayyip Erdoğan ihre Außenpolitik neu ausgerichtet. Der EU-Beitritt hat an Priorität verloren. Das Ziel der neuen türkischen Außenpolitik ist die Etablierung der Türkei als Regionalmacht, die vorwiegend auf der regionalen Bühne aktiv ist. So spielt die Türkei in vielen regionalen Konflikten eine Vermittlerrolle. Das ist eine fundamentale Abkehr der türkischen Außenpolitik von ihrer zentralen Ausrichtung auf Europa und den Westen. Die Identität der Türkei ist nicht länger nur europäisch beziehungsweise strebe nicht länger nur nach einer solchen Identität, sondern verfüge über multiple Identitäten, wie der türkische Außenminister betont. Diese Neuausrichtung ist unter anderem deshalb erfolgt, weil innerhalb der türkischen Regierung Zweifel an der Aufnahme der Türkei in die Europäische Union bestehen.

Es gibt drei Modelle, die am Ende von erfolgreichen Beitrittsverhandlungen stehen können: erstens, die Mitgliedschaft; zweitens, die Verweigerung der Mitgliedschaft durch die Europäische Union bei einem Aufrechterhal- tenen des türkischen Beitrittswunsches (, britisches Modell'); und drittens die Verweigerung des Beitritts durch die Türkei nach erfolgreich abgeschlossenen Verhandlungen (,norwegisches Modell'). Das , norwegische Modell ' würde gut in die neue Außenpolitik der Regierung Erdoğan passen.

Ersin Kalaycıoğlu begann seinen Vortrag mit einem Witz darüber, wie die türkische öffentliche Meinung die Beitrittsverhandlungen wahrnimmt (Perzeption doppelter Standards): Bulgarien, Rumänien und die Türkei treten vor die Europäische Union, die über einen Beitritt befindet. Als erstes wird Bulgarien gefragt, welches Ereignis den Zweiten Weltkrieg beendete. Bulgarien antwortet: „Der Abwurf der Atombombe auf Japan.“ Die Europäische Union ist zufrieden und nimmt Bulgarien auf. Als nächstes wird Rumänien gefragt, wo die erste Atombombe abgeworfen wurde. Rumänien antwortet: „Hiroshima“. Die Europäische Union ist erneut zufrieden und nimmt Rumänien ebenfalls auf. Schließlich ist die Türkei an der Reihe und wird gefragt, wo die zweite Atombombe abgeworfen wurde. Die Türkei antwortet: „Nagasaki“. Die Europäische Union sagt: „Sehr gut. Jetzt nenne bitte noch die Namen aller Opfer und ihrer Angehörigen." Nach diesem einführenden Witz, der verdeutlicht, wie die türkische Bevölkerung die EU-Beitrittspolitik gegenüber der Türkei empfindet, sprach Kalaycıoğlu über die Einstellungen der Türken zur Moderne und zu Europa.

47 Prozent der türkischen Bevölkerung möchten zurück zu ihren alten Werten, den Werten der türkischen Agrargesellschaft. Nur 20 Prozent befürworten weitere liberale Reformen. 50 Prozent der Türken sprechen sich für eine Mitgliedschaft in der Europäischen Union aus, 37 Prozent sind gegen eine EU-Mitgliedschaft. 71 Prozent der Türken äußern die Meinung, die Türkei kann ihre Probleme alleine lösen und braucht dafür kein Mitglied der Europäischen Union zu werden. 18 Prozent der türkischen Bürger sind der Meinung, dass die Türkei ihre Probleme nur durch eine EU-Mit- 
gliedschaft wird lösen können (alle Zahlen von 2007). 2009 sind die Werte vergleichbar: 56 Prozent der Türken sind für einen EU-Beitritt und 32 Prozent sind dagegen. 65 Prozent meinen, die Türkei kann ihre Probleme alleine lösen und 23 Prozent denken, dass die Türkei ihre Probleme nur durch eine EU-Mitgliedschaft wird lösen können. Die Zustimmungsraten für einen EU-Beitritt schwanken im Zeitraum 2002 bis 2009 zwischen 50 und 70 Prozent. Die Zustimmungsrate ist relativ konstant über die Zeit.

Haupterklärungsfaktor für eine Zustimmung oder Ablehnung eines Beitritts ist die Parteizugehörigkeit. So sind Anhänger der Regierungspartei AKP überwiegend für einen EUBeitritt und Anhänger der Oppositionspartei CHP überwiegend gegen einen Beitritt, weil die jeweiligen Parteien einem Beitritt positiv beziehungsweise skeptisch gegenüber stehen. Religiosität, Ideologie oder sozioökonomischer Status bieten hingegen keine Erklärung für den Grad der Zustimmung einer Person. Einzig Frauen und Kurden sind etwas stärker für einen EU-Beitritt als Männer und Nichtkurden.

Bei der Zypernfrage betonte Kalaycıŏlu die türkische Position: Warum sollte die Türkei sich um eine Lösung der Zypernfrage bemühen und Konzessionen machen, wenn sie im Gegenzug keine Garantie für eine Mitgliedschaft in der Europäischen Union bekommen wird? Die Europäische Union verlangt von der Türkei unter anderem die Aufhebung eines Embargos gegen griechisch-zypriotische Schiffe in türkischen Häfen. Die griechischzypriotischen Schiffe benutzen de facto bereits türkische Häfen, indem sie bei Einfahrt in den türkischen Hafen die griechische
Flagge aufziehen. Damit gibt es de facto kein Embargo der türkischen Regierung gegen griechisch-zypriotische Schiffe. Die türkische Regierung möchte die Häfen aus innenpolitischen Gründen nicht öffnen, weil die Regierung dann Stimmen an die nationalistische Opposition verlieren würde. Die Zypernfrage kann nur gelöst werden, wenn die griechischen Zyprioten den UN-Plan ${ }^{1}$ zur Wiedervereinigung akzeptieren. Als EU-Mitgliedstaat kann sie aber niemand dazu zwingen.

Weiterhin besteht ein großes innenpolitisches Problem in der Türkei in der mangelnden Legitimität der Regierung und der Opposition. Die türkische Regierung wurde vom Obersten Gerichtshof für schuldig befunden, die Säkularität der Türkei untergraben zu haben. Damit hat die Regierungspartei gegen einen zentralen Grundsatz der türkischen Verfassung verstoßen. Die Opposition ist antidemokratisch und wird beschuldigt, einen Putsch gegen die Regierung geplant zu haben (Ergenekon-Affäre). Regierung und Opposition verfügen somit beide über eine unzureichende Legitimität. Die Demokratie in der Türkei und die politische Entwicklung stecken damit in einer Sackgasse, die vor den nächsten Wahlen im Jahr 2011 nicht aufgelöst werden kann.

Insgesamt hat die Tagung die vielfältigen Chancen und Herausforderungen eines türkischen EU-Beitritts beleuchtet. Die Tagung hat gezeigt, dass viele weitere türkisch-europäische Kooperationsprojekte notwendig sind, um die Türkei weiter an Europa und Europa weiter an die Türkei anzunähern und gegenseitige Vorbehalte abzubauen.

1 Der sogenannte Annan-Plan: The Comprehensive Settlement of the Cyprus Problem. The documents appended constitute the Comprehensive Settlement of the Cyprus Problem as finalised on 31 March 2004, abrufbar unter: http://www.unficyp.org/media/Other\%20official\%20documents/annanplan.pdf (letzter Zugriff: 26.11.2009). 